\title{
L'Unification des Forces en Physique: Les Premières Tentatives*
}

\author{
JOSÉ LEITE LOPES** \\ Centro Brasileiro de Pesquisas Físicas - CBPF \\ Rua Dr. Xavier Sigaud, 150 \\ 22290-180 - Rio de Janeiro, RJ, Brasil
}

Manuscript received on March 30, 2000; accepted for publication on April 3, 2000.

\begin{abstract}
The author gives a brief description of the studies that led to the eclosion of the weak forces model of unification of electromagnetic interactions with weak interactions.
\end{abstract}

Key words: weak forces model, unification of interactions, neutral Boson vectorial.

Les hommes de science qui prennent une part active dans les recherches de frontière de leur spécialité savent bien que les grandes découvertes ne sont pas le résultat de travaux de chercheurs à une époque donnée - elles sont plutôt une conséquence de travaux effectués quelques années avant leur éclosion. Beaucoup plus tard, aprés des décennies et de siècles, ce qui reste, en histoire de la science, c'est la découverte associée au nom d'un ou de quelques scientifiques; les contributions importantes qui les ont précédées, sont laissées de côté, oublieés. Il est clair que l'homme de génie est celui qui voit dans l'assembleé des ideés qui se discutent à une époque donnée celles qui débouchent dans une nouvelle conception - et il la met en lumière et la developpe - ouvrant des nouveaux horizons dans son domaine. P.A.M. Dirac [1] a exprimé cette façon de voir l'histoire de la science d'une façon précise: "When one looks over the development of physics, one sees that it can be pictured as a rather steady development with many small steps and superposed on that a number of big jumps. Of course, it is these big jumps which are the most interesting features of this development. The background of steady development is largely logical, people are working out the ideas which follow the previous set-up according to standard methods. But then when we have a big jump, it means that something entirely new has to be introduced. These big jumps usually consist in overcoming a prejudice".

\footnotetext{
*Article invité

** Membre de l' Academia Brasileira de Ciências
} 
Néanmoins, la tendance moderne des historiens de la science semble être de montrer que chaque phase historique regardée comme essentiellement différente de phases précédentes n'était en realité que le résultat de l'action continue de grandes forces sousjacentes agissant continuellement.

"Le vieux style" affirme Stillman Drake [2], était de montrer chaque scientifique pionnier comme un révolutionnaire, qui reconnaissait sa dette au passé aussi peu que possible et qui soulignait la nouveauté de son travail autant que possible. Le style actuel est d'attribuer sa pensée autant que possible à ses predecesseurs et de lui accorder sa propre originalité aussi peu que possible". C'est un style que Drake veut complété par une étude approfondie de la vie des auteurs de grandes découvertes.

Pour bien comprendre l'histoire de la théorie des champs de jauge unifiés il faut remonter au fameaux papier de Enrico Fermi [3], de 1934, sur une tentative de théorie des rayons beta.

Ce fut la première application important des idées qui venaient d'être developpées en électrodynamique quantique, notamment par P.A.M. Dirac, W. Heinsenberg, W. Pauli, P. Jordan, E.P. Wigner et par Fermi lui même [4]. Dans l'article de Fermi il affirme que, d'après la théorie du rayonnement électromagnétique, le nombre de photons dans un système n'est pas constant; les photons sont créés lorsqu'ils sont émis par un atome, ils disparaissent lorsqu'ils sont absorbés. Ainsi dans sa théorie de la désintegration beta il postule que "le nombre d'electrons aussi bien que celui de neutrinos n'est pas necessairement constant. Electrons (ou neutrinos) peuvent être créés ou détruits”. Le noyau étant regardé comme constitué de protons et neutrons [5], Fermi dit que l'hamiltonien doit être exprimé en fonction des variables des nucleons et des leptons et choisi de telle façon que chaque transition d'un neutron dans un proton doit être associeé avec la création d'un electron et d'un neutrino (aujord'hui, on le sait, c'est l'anti-neutrino qui accompagne l'electron dans des réactions où le nombre leptonique est null).

La préocupation de Fermi était de décrire les experiences sur les rayons beta émis par les noyaux et par conséquent sa théorie avait pour but de décrire des electrons et des neutrinos créés et qui se propagent librement comme les photons dans l'émission de la lumièrè. Il a donc remplacé le champ électromagnétique $A_{\mu}(x)$ dans le lagrangéen d'interaction de ce champ avec le courant électromagnétique

$$
J^{\mu}(x)=\bar{\Psi}(x) \gamma^{\mu} \Psi(x)
$$

à savoir

$$
L_{\gamma}=e\left(\bar{\Psi}(x) \gamma^{\mu} \Psi(x)\right) A_{\mu}(x)
$$

par l'expression qui décrit la création d'un électron et d'un anti-neutrino - le courant faible chargé leptonique de la famille electron - à savoir $\bar{e}(x) \gamma_{\mu} v(x)$. Ainsi donc si $\frac{G}{\sqrt{2}}$ est la constante qui remplace dans cette théorie la charge $\underline{e}$ et qui exprime l'intensité des interactions faibles, Fermi a postulé le lagrangeen de sa théorie des rayons $\beta$ :

$$
L_{\mathrm{W}}=\frac{G}{\sqrt{2}}\left(\bar{p}(x) \gamma^{\mu} n(x)\right)\left(\bar{e}(x) \gamma_{\mu} \nu(x)\right)
$$


L'analogie avec l'électrodynamique l'a incité à choisir l'interaction vectorielle.

En même temps que Fermi, F. Perrin [6] publiait également son article sur le même sujet, en suggerant essentiellement les mêmes idées. Plusieurs auteurs [7] ont, juste aprés la publication du papier de Fermi, étudié la possibilité que les paires electron-anti-neutrino, échangées entre un neutron et un proton, donneraient lieu à une interaction neutron-proton, similaire à l'interaction électromagnétique entre les corpuscules chargés qui est décrite par un échange de photons (virtuels) entre ces corpuscules. Cette conception néanmoins ne conduit pas à une description de l'interaction forte entre un neutron et un proton tout d'abord parce que l'interaction ainsi considéreé serait trop faible. Ce fut alors en 1935 que H. Yukawa [8] introduisit l'ideé d'un boson intermédiaire, qui devrait être échangé entre les nucleons et qui engendrerait ainsi l'interaction nucléaire. Yukawa determina l'ordre de grandeur de la masse de ce boson en prenant en compte la portée des forces nucléaires. À cette epoque là on évitait d'introduire de nouvelles particules hypothétiques, c'est pourquoi le papier de Yukawa ne commença à etre étudié qu'aprés la découverte de particules avec cette masse dans la radiation cosmique par S.H. Neddermeyer et C.D. Anderson [9]. Il s'avera plus tard [10], que les particules de Yukawa sont les pions, avec spin zero, tandis que les particules de Anderson et Neddemeyer sont les muons avec spin 1/2. Yukawa voulait que sa theorie fût capable de décrire aussi bien les interactions nucléaires que les interactions faibles - le boson negatif de Yukawa, émis par un neutron dévrait ensuite se désintegrer dans un electron et un anti-neutrino. Cette conception unifieé ne put pas être maintenue [11].

Le manque de connaissance de la forme géométrique précise des interactions faibles empecha pendant longtemps la considération de bosons intermédiaires comme les véhicules de ces interactions. Ce ne fut que par les papiers de E.G.C. Sudarshan et R.E. Marshak, d'une part, et de R.P. Feynman et M. Gell-Mann, d'autre part - et aussi de J. Sakurai [12] - que la forme de l'interaction faible fut découverte comme une combinaison des formes vectorielle V et axiale A, à savoir, V-A. Dans leur article Feynman et Gell-Mann disent:

"Nous avons adopté le point de vue selon lequel toutes les interactions faibles resultent de l'interaction d'un courant $J_{\mu}$ avec lui-même, possiblement par l'intermédiaire de mesons vectoriels de masse large".

Ainsi l'ideé de bosons vectoriels intermédiaires dans les interactions de Fermi s'est montrée possible malgré les difficultés de ce modèle: comme dans l'année 1958 on ne savait pas de l'existence de neutrinos muoniques, différents des neutrinos électroniques, G. Feinberg [13] a indiqué que l'absence de la desintégration radiative du muon, $\mu \rightarrow e+\gamma$, est incompatible avec la théorie des bosons vectoriels intermédiaires.

En cette même année de 1958, en lisant l'article de Feynman et Gell-Mann j'ai tout de suite eu le sentiment que si les interactions faibles etaient dues à l'échange de bosons vectoriels intermédiaires, elles devraient être intimement relationnées avec les interactions électromagnétiques, transmises par des photons qui sont eux aussi des particules vectorielles. Une idée d'unification, je l'ai proposée [14] en admettant que l'intensité des interactions électromagnétiques $\underline{e}$ avec les courants 
chargés est égale à celle des interactions faibles avec les courants correspondants, $\underline{g}$, une idée qui est implicite dans cette égalité, et dans la même nature géometrique des photons et des bosons intermédiaires. Si l'on remplace $\underline{g}$ par $\underline{e}$ dans la rélation entre la constante de Fermi G et la masse de ces bosons, $m_{\mathrm{W}}$, on obtient une valeur de cette masse de l'ordre de 60 fois la masse du proton. C'était la prémière fois qu'on donnait une valeur de l'ordre de grandeur de la valeur de la masse $m_{\mathrm{W}}-$ deux années plus tard, T.D. Lee et C.N. Yang [15] indiquaient simplement que $m_{\mathrm{W}}$ devrait être superieure à $m_{k}$, la masse du kaon, afin de justifier l'absence de la désintégration radiative $K^{ \pm} \rightarrow W^{ \pm}+\gamma$. Et d'après Pontecorvo [16] "in 1959 the intermediate boson (without serious reasons) was supposed to have a mass of a few GeV”. Plus tard, en 1971, par des considerations d'algèbre des courants, T.D. Lee [17] proposa une évaluation de la masse $m_{\mathrm{W}}$, en arrivant à une rélation de la forme $e=2 \sqrt{2} g$. L'algèbre des courants et le postulat d'un lagrangean avec la même forme pour les interactions faibles et pour les interactions électromagnétiques conduisent à une relation [18]

$$
e=\frac{2 \eta}{\sqrt{2}} g
$$

où $\eta$ est un facteur numérique. Selon les valeurs de $\eta$ on obtient l'egalité $e=g$ ou la rélation de Lee, $\eta=2$, ou la rélation de Weinberg, $\eta=\frac{\sqrt{2}}{2} \sin \theta_{\mathrm{W}}$, où $\theta_{\mathrm{W}}$ est l'angle de Weinberg. C'est la dernière formule celle qui est predite par le modèle standard et qui est d'accord avec l'experience [19].

La valeur de la masse des bosons vectoriels intermédiaires posait le problème suivant: comment considerer les photons et les bosons $W$ comme membres d'un multiplet s'il y a une telle difference de masse entre eux? C'est le même problème qui se pose formellement lorsqu' on décrit ces bosons par des champs de Yang-Mills dans une théorie avec invariance de jauge. Cette invariance exige pour ces champs, comme pour le champ électromagnétique, une masse nulle, ce qui n'est pas compatible avec l'estimation de masse ci-dessus - et ce problème, comme on le sait, n'a été résolu que par la découverte du mécanisme de Higgs [25].

Une fois qu'on devrait prendre au serieux l'ideé que les interactions faibles étaient dues à un echange de bosons vectoriels comme je l'ai proposé dans mon article de 1958, la question apparaissait de savoir s'il n'existerait pas également des interactions faibles dues à un échange de bosons vectoriels neutres. On savait, en théorie du champ mesonique des forces núcleaires, de l'existence de pions neutres et surtout que l'invariance du lagrangéen par rapport au groupe SU(2) entraîne la même forme et la même intensité, pour les interactions au moyen de pions chargés comme pour celles mettant en jeu des pions neutres. J'ai alors proposé un modèle en postulant l'existence des bosons vectoriels intermédiaires et chargés et neutres; pour éliminer des transitions non-observeés l'hypothèse a été faite que le courant vectoriel neutre devrait être conservé - et la théorie serait alors dependente de charge (à la difference du cas pionique). Comment verifier experimentalement l'existence des possibles bosons vectoriels neutres? Puisque des faisceaux de neutrinos n'étaient pas encore realisés experimentalement, j'ai suggeré [14] que l'étude de 
la diffusion de neutrons par des électrons pourrait donner une indication de l'existence d'une interaction faible de courants neutres. Les bosons neutres $Z$, comme on le sait, étaient aussi prédits neuf ans plus tard par Steve Weinberg [20] et le modèle standard donne une valeur pour les masses $m_{\mathrm{W}}$ et $m_{z}$ en accord avec les mésures au laboratoire [21].

Les papiers de J. Schwinger et de S. Bludman [22] ont introduit la conception selon laquelle les interactions faibles comme les interactions électromagnétiques devraient obeir à un principe d'invariance par rapport à un groupe local de symétrie. En particulier, Bludman introduisit les interactions mettant en jeu les courants neutres faibles. Sheldon Glashow aussi bien que A. Salam et J. Ward [23] ont également proposé des tentatives d'unification des interactions faibles et electromagnétiques et les courants neutres qu'ils ont introduit avaient des propriétés communes avec celles du modèle standard. Comme l'experience jusqu'à l'année 1971 n'avait pas encore indiqué l'existence des courants faibles neutres, H. Georgi et Glashow [23] ont proposé un modèle ingenieux, basé sur la symétrie SU(2), selon lequel les champs de jauge seraient les champs électromagnétiques et ceux qui correspondent aux bosons vectoriels chargés. Les deux articles qui ont finalement établi les bases du modèle standard basé sur le groupe $S U(2) \otimes U(1)$ sont ceux de Weinberg [20] en 1967 et de Salam [24] en 1968. La difficulté de la génération de la masse des bosons chargés et neutre, comme on le sait, a été resolue par la notion de symetrie spontanement brisée qui a donné lieu au mécanisme de Higgs [25]. Et la théorie s'est reveleé, comme l'a antecipé Weinberg, renormalizable [26].

Ainsi, si le développement du modèle standard d'unification des forces faible et électromagnétique est dû aux travaux de Weinberg, Glashow et Salam, il est également vrai que certaines tentatives qui les précéderent ont contribué à dévoiler les idées fondamentales de cette belle théorie.

J'aimerais dédier cet article à la memoire de Carlos Chagas Filho.

\section{REFERENCES}

[1] Dirac PAM. in Jadish Mehra (editeur) 1973. The physicist's conception of nature page 10, D. Reidel Publ. Co., Dordrecht.

[2] Stillman Drake. 1970. Galileo Studies, personality, tradition and revolution. The University of Michigan Press, An Arbor.

[3] E. Fermi. 1934. Zeit. f. Phys. 88: 161.

[4] VoIr J. Schwinger. Quantum electrodynamies.

[5] D. Iwanenko, C.R. Acad. Sci. Paris 195: 439 (1932); W. Heisenberg, Zeit. f. Phys. 77: 1 (1932).

[6] F. Perrin, C.R. Acad. Sci. Paris 197: 1625 (1933).

[7] I.G. TAмм, Nature (London) 133: 982 (1934); D. IwanenKo, Nature (London) 133: 981 (1934); G.C. WICK, Rend. Lincei 21: 170 (1935). 
[8] H. Yukawa, Proc. Phys. Math. Soc. Japan 17: 48 (1935).

[9] C.D. Anderson \& S.H. Neddermeyer, Phys. Rev. 51: 884 (1937).

[10] O. PiCcioni in Coloque International sur l'histoire de la physique des particules, Journ. de Physique, Tome 43, Colloque C8, C8-207 (1982); C.M.G. Lattes, G.P.S. Occhialini \& C.F. Powell, Nature 160: 453 (1947).

[11] M. Ruderman \& R. Finkelstein, Phys. Rev. 76: 1458 (1949); J. Leite Lopes, Phys. Rev. 109: 509 (1958).

[12] E.G.C. Sudarshan \& R.E. Marshak, Proc. Padua Conf. on mesons and recently discovered particles v.14 (1957); R.P. Feynman \& M. Gell-Mann, Phys. Rev. 109: 193 (1958); J. Sakurai, Nuovo Cimento Z, 649 (1958).

[13] G. Feinberg, Phys. Rev. 110: 1482 (1958).

[14] J. Leite Lopes, Nucl. Phys. 8: 234 (1958).

[15] T.D. Lee \& C.N. Yang, Phys. Rev. 119: 1410 (1960); voir C.N. Yang, Selected papers with commentary, W.H. FreEman, San Francisco (1983).

[16] B. Pontecorvo in Colloque International sur l'histoire de la physique des particules, Journ. Physique Tome 43 Colloque C8, C8-221 (1982).

[17] T.D. LEE, Phys. Rev. 26: 801 (1971).

[18] J. LeIte Lopes in Physical Reality and Mathematical Description. Enz/Mehra (editeurs), 403, D. Reidel Publ. Co. Dordrecht (1974).

[19] P. Musset \& J.P. Vialle, Phys. Rep. 39C: 1 (1978).

[20] S. Weinberg, Phys. Rev. 19: 1264 (1967).

[21] UA1 Collaboration CERN Phys. Letters 126: 398 (1983); 135: 250 (1983); UA2 Collaboration CERN Phys. Letters B129: 130 (1983).

[22] J. Schwinger, Ann. Phys. 2: 407 (1957); S. Bludman, Nuovo Cimento 9: 433 (1958).

[23] S. Glashow, Nucl. Phys. 22: 579 (1961); A. Salam \& J. Ward, Phys. Letters 13: 168 (1964).

[24] A. Salam, Nobel Symposium, Ed. Swartholm. Almqvist and Wiksell, Stockholm (1968).

[25] Les contributions sur la brisure spontánee de symétrie furent essentielles à la théorie: J. GoLDSTONE, Nuovo Cimento 19: 154 (1961); P.W. Higgs, Phys. Lett. 12: 132 (1964); F. Englert \& R. Brout, Phys. Rev. Lett. 13: 321 (1964); G.S. Guralnik, C.R. Hagen \& T.W. Kibble, Phys. Rev. Lett. 13: 585 (1965).

[26] G’т Hooft. 1971. Nucl. Phys. B33: 173. 